\title{
Éjaculation prématurée Avec la collaboration de la Société Francophone de Médecine Sexuelle (SFMS)
}

\section{Premature ejaculation}

(C) SALF et Springer-Verlag France 2010

\author{
Vers un retour à la section et plastie du frein \\ dans des cas sélectionnés?

\section{The role of short frenulum and the effects of frenulectomy on premature ejaculation} \\ Gallo L, Perdonà S, Gallo A (2010) J Sex Med 7:1269-76 \\ M. Galiano
}

Il s'agit d'une étude originale qui relance l'intérêt de la section plastie du frein comme première ligne dans le traitement de l'éjaculation précoce. Quarante patients ayant comme motif de consultation une éjaculation précoce ont eu un diagnostic de frein court. Le critère de jugement était une angulation d'au moins $20^{\circ}$ entre le gland et le fourreau suite à une traction modérée mais complète du prépuce. Ce critère n'a fait l'objet d'aucune publication mais a le mérite de paraître logique et acceptable du moment où l'on considère que la traction du frein ne devrait pas entraver les mouvements du gland. L'intervention s'est déroulée dans la majorité des cas sous anesthésie locale topique, et le frein a été totalement sectionné et sa partie glandulaire coagulée. La reconstruction s'est faite par des points séparés.

Cette étude ouverte a mis en évidence de façon significative une amélioration du temps d'éjaculation intravaginale qui était de 1,65 minute en moyenne en préopératoire et de 4,11 minutes en postopératoire, ainsi qu'une diminution de 5,95 points en moyenne du score symptôme (PEDT).

Les auteurs en s'appuyant sur la littérature argumentent l'intérêt de cette technique dans le traitement de l'éjaculation précoce. En effet, un frein court peut être une épine irritative source de douleur et d'une hyperstimulation des zones thalamiques et corticales qui, en retour, lèvent l'inhibition des segments lombaires médullaires responsables de la phase d'expulsion. Par ailleurs, l'innervation propre du frein provenant des branches sensitives du nerf pudendal, sa section ne diminue que partiellement les zones érogènes du gland et ne serait donc pas une contreindication à sa destruction.

On peut simplement faire comme commentaire que tous les patients qui ont une section du frein involontaire par accident ou volontaire par l'urologue ne se plaignaient pas tous d'éjaculation précoce mais que l'absence de frein qui s'en est suivie n'a en rien augmenté leur capacité à différer leur éjaculation. Ou tout au moins, cela n'a été ni recherché ni rapporté. Cet effet bénéfique de la section du frein mérite d'être vérifié dans d'autres études.

En conséquence, nous pouvons élaborer un mini-arbre décisionnel qui serait que tout patient souffrant d'éjaculation précoce doit avoir un examen clinique de la verge et qu'un frein court (une angulation de $20^{\circ}$ lors d'une traction du prépuce peut être retenue jusqu'à preuve du contraire) pourrait faire l'objet d'une chirurgie en cas d'absence d'amélioration ou d'échec des techniques sexologiques comportementales mais avant l'instauration d'un traitement médical chronique. Un frein normal pourrait faire l'objet d'une prise en charge uniquement médicosexologique, dont les modalités et la chronologie resteraient à débattre. Enfin, il serait également intéressant, avec l'arrivée prochaine de la dapoxetine, de réaliser une étude randomisée entre une chirurgie simple et rapide et le traitement médical à la demande.

J. Buvat $(\bowtie)$

CETPARP, 3, rue Carolus, F-59000 Lille, France

e-mail : Jacques@buvat.org 
La saga du déterminisme génétique de l'éjaculation prématurée se poursuit avec un possible rôle du polymorphisme du gène du transporteur de la dopamine

\section{The dopamine transporter gene (DAT1) polymorphism is associated with premature ejaculation}

Santtila P, Jern P, Westberg L, et al (2010) J Sex Med $7: 1538-46$

\section{J. Buvat}

Introduction : La possibilité d'une composante héréditaire dans le déterminisme de l'éjaculation prématurée (EP) a été proposée dès 1943 par Schapiro. Elle a été confirmée en 2009 par les travaux chez les jumeaux de Jern et Santtila (voir bulletin électronique de la SFMS $\mathrm{n}^{\circ} 3$, juin 2009). Depuis 18 mois, plusieurs travaux du même groupe finnois, ainsi que ceux du groupe de Waldinger (voir bulletin SFMS $\mathrm{n}^{\circ} 4$, juillet 2009) et du groupe iranien de Safarinejad ont particulièrement rapporté des corrélations entre EP, ou délai éjaculatoire, et présence de certains variants polymorphiques du gène du transporteur de la sérotonine.

Dans l'étude analysée, c'est au gène du transporteur de la dopamine (DA) que se sont intéressés les auteurs finnois. Les effets modulants de la DA sur le comportement sexuel masculin ont été démontrés dès les années 1970. On peut résumer les données disponibles en disant que, dans l'ensemble, la régulation dopaminergique centrale semble facilitante tant pour l'érection que pour l'éjaculation. Dans l'espèce humaine, des études en PET-scan ont montré que l'éjaculation induite au cours d'une relation sexuelle activait des régions cérébrales riches en DA, régions habituellement associées avec les comportements de récompense telles que la prise de cocaïne ou d'héroïne. Le transporteur de la DA est une protéine responsable de la recapture de la DA par le neurone présynaptique, réduisant de ce fait la quantité de DA disponible pour se lier aux récepteurs dopaminergiques dans la synapse. Le polymorphisme de ce gène s'exprime au niveau du nombre de répétitions « tandem », variant de 3 à 11. Les variants comportant neuf et dix répétitions (respectivement $9 R$ et $10 R$ ) sont les plus fréquents. Les études en résonance magnétique nucléaire et en PET-scan suggèrent que l'allèle 9R est associé avec une augmentation du tonus dopaminergique et une augmentation de l'activation des régions cérébrales à innervation dopaminergique.

Patients et méthodes : Cette étude a porté sur 1290 Finnois de 18 à 33 ans, dont 867 étaient jumeaux et les autres frères de ces jumeaux. Les analyses de l'ADN ont été effectuées à partir d'échantillons de salive, et l'évaluation de la fonction éjaculatoire a été basée sur des questions évaluant la proportion des rapports au cours desquels survenait une éjaculation ante portas, le nombre maximum de mouvements après lesquels l'éjaculation survenait, le sentiment de contrôler l'éjaculation et enfin le temps de latence éjaculatoire. Après extraction de l'ADN génomique de la salive, une partie de la région 3'UTR a été amplifiée par PCR, et les fragments d'ADN marqués par fluorescence ont été analysés en fonction de leur taille par électrophorèse capillaire automatisée.

L'analyse des données a consisté à rechercher des corrélations entre les paramètres d'éjaculation et le type de variant polymorphique (9R ou $10 \mathrm{R}$ ), après ajustement des données en fonction de l'âge, de l'existence d'une partenaire sexuelle régulière, d'une éventuelle homosexualité, du niveau de désir sexuel et de la fréquence des différents types d'activité sexuelle.

Résultats : Par rapport aux porteurs du génotype 9R (soit homozygote, 9R/9R, soit hétérozygote, 9R/10R), les porteurs homozygotes du génotype 10R avaient un seuil éjaculatoire plus bas selon chacun des paramètres évalués. Les différences étaient statistiquement significatives à la fois pour le score composite d'EP, pour l'EP ante portas, pour le nombre de mouvements pelviens avant éjaculation et pour le sentiment de contrôle de l'éjaculation mais pas pour le temps de latence éjaculatoire. Cet impact du polymorphisme sur l'éjaculation restait statistiquement significatif après ajustement des données en fonction des paramètres précisés dans le paragraphe précédent, suggérant qu'il n'était pas secondaire à une association entre le polymorphisme et d'autres aspects du comportement sexuel, mais au contraire dû à une influence spécifique de la DA sur l'éjaculation.

Discussion : Les résultats de cette étude suggèrent que les individus homozygotes pour le variant $10 \mathrm{R}$ du gène du transporteur de la DA ont un risque significativement plus élevé d'être affectés par une EP. Cet effet paraît indépendant du niveau de désir sexuel, alors que ce variant $10 \mathrm{R}$ a été trouvé corrélé dans une autre étude au fait d'avoir un nombre plus élevé de partenaires sexuels. Il est à noter que cette formule $10 \mathrm{R} / 10 \mathrm{R}$ est la plus fréquente (environ $65 \%$ de la population). Qu'elle soit corrélée à un seuil plus bas de déclenchement de l'éjaculation peut être un argument en faveur de la théorie selon laquelle éjaculer rapidement donnait par le passé plus de chances à l'individu d'échapper aux prédateurs au cours de son coït ou d'éjaculer avant les attaques des autres mâles compétiteurs, conduisant logiquement à ce que ce génotype soit plus représenté dans la population générale.

Les résultats de cette étude inciteront peut-être à tester de nouveau les antagonistes dopaminergiques dans le traitement de l'EP. Il faut rappeler que certains des tout premiers médicaments psychotropes prescrits pour améliorer l'EP étaient des antidopaminergiques, tout particulièrement la thioridazine $\left(\right.$ Melleril ${ }^{\circledR}$ ) et que les antidopaminergiques font partie des principaux pourvoyeurs de troubles sexuels iatrogènes et particulièrement d'anéjaculation. 
Faut-il enfin rappeler qu'il n'est bien sûr pas question de réduire l'étiopathogénie de l'EP à un déterminisme purement génétique ? Les travaux du groupe de Jern et Santtila ont bien montré que le facteur génétique ne représentait qu'environ $30 \%$ de la variance des cas d'EP qu'ils ont évalués dans leurs études, et que des facteurs éducatifs psychologiques et liés à la partenaire jouaient certainement également un rôle important (voir bulletin électronique de la SFMS $n^{\circ} 3$, juin 2009). Mais on imagine très bien que les effets d'une prédisposition biologique sur l'éjaculation puissent être modulés par les particularités de la personnalité de l'individu, par son environnement socioéducatif, ainsi que par celles de sa relation avec sa partenaire. Mais il peut être important de prendre en considération la possibilité d'un tel facteur biologique favorisant, particulièrement dans l'EP primaire. Par ailleurs, l'accumulation récente d'études ayant mis en évidence un possible rôle de plusieurs types de polymorphisme de gènes codant pour des neurotransmetteurs ou pour leur transporteur est également en accord avec l'hypothèse de Waldinger. Celui-ci pense en effet que ce sont des interactions entre plusieurs particularités génétiques présentes chez un même individu qui constituent le facteur biologique favorisant l'EP primaire, et non pas un seul variant polymorphique.

\section{Testostérone}

Chez l'homme un taux faible de testostérone est associé à une augmentation de la consommation médicale

\section{Prospective association of low serum total testosterone levels with health care utilization and costs in a population-based cohort of men}

Haring R, Baumesiter SE, Völzke H, et al (2009) Int J Androl 33:1-10

\section{J. Buvat}

Patients et méthodes : Il s'agit de la première étude qui ait évalué l'impact des taux de testostérone circulante sur l'utilisation des services de santé et les coûts en résultant. Elle est basée sur l'analyse des données de la cohorte allemande de la Study of Health In Pomerania (SHIP). Lors de l'inclusion dans l'étude, la cohorte comprenait 2023 hommes (20 à 79 ans), parmi lesquels 1530 ont été revus cinq ans plus tard. Ont été qualifiés de taux respectivement bas et élevés les taux de testostérone plasmatique situés en dessous du $10^{\mathrm{e}}$ et au-dessus du $90^{\text {e }}$ percentile spécifiques de l'âge du patient. Différents modèles socio-économiques et médicaux ont été appliqués. Résultats : Les analyses transversales ont trouvé des chiffres plus élevés de visites dans des services de santé ambulatoire, et des coûts de santé plus élevés en rapport avec ces visites, chez les hommes avec testostérone basse (respectivement $+19,1$ et $+19,9 \%$ ) mais aussi chez ceux avec testostérone élevée (respectivement $+25,3$ et $+30,2 \%$ ). Par contre, ni le nombre de jours d'hospitalisation ni les coûts en résultant ne différaient en fonction du taux de testostérone. L'ajustement des données pour l'âge, le niveau éducatif, le niveau du revenu, le tour de taille, le fait de fumer, l'activité physique et la consommation d'alcool n'a pas modifié les résultats de façon importante. Les analyses longitudinales ont trouvé une association significative entre taux de testostérone bas et augmentation du nombre des visites de suivi dans les services de santé ambulatoires ( $+26 \%$ après ajustement pour l'âge), ainsi qu'entre testostérone basse et coûts de santé $(+38 \%)$, mais n'ont par contre plus trouvé ce type de corrélations chez les sujets à taux élevé de testostérone.

Conclusion : Le fait d'avoir une testostérone basse ou élevée était dans cette étude corrélé à une augmentation des coûts de santé ambulatoire à court terme, mais seuls les taux de testostérone bas paraissaient prédictifs d'une augmentation des coûts de santé ambulatoire à long terme. Des études portant sur la rentabilité des traitements par la testostérone semblent souhaitables afin de préciser le bénéfice que pourraient en tirer médecins, patients et ensemble du système de santé.

Discussion : Les auteurs rappellent que les corrélations entre taux faible de testostérone et facteurs de risque cardiovasculaire sont bien connues, mais que cette relation est complexe et pas obligatoirement causale. Il apparaît aujourd'hui qu'elle fonctionne dans les deux sens, les taux faibles de testostérone prédisposant à l'obésité viscérale, tandis que l'obésité viscérale inhibe la production de testostérone. Cela doit d'autant plus être pris en compte dans leur étude que la Poméranie est une région où la prévalence des comportements à risque (consommation de tabac et d'alcool), comme celles des maladies chroniques que sont l'obésité, l'hypertension, le syndrome métabolique et le diabète (qui peuvent toutes augmenter la morbidité et la mortalité) sont particulièrement importantes.

Ils discutent également la corrélation trouvée dans l'analyse transversale de leurs données entre taux de testostérone élevé et augmentation de l'utilisation des structures de soins ambulatoires, de façon indépendante des facteurs confondants classiques. Plusieurs études ont trouvé des corrélations entre taux de testostérone élevé et comportement de prise de risques, ou de recherche de sensations fortes, dont on sait qu'ils sont corrélés à des risques pour la santé. Mais dans le cas présent, les études longitudinales n'ont pas confirmé cette corrélation transversale initiale dont la signification n'est donc probablement que relative.

En ce qui concerne les analyses de sensibilité, les auteurs ont exclu de l'échantillon analysé les 241 sujets décédés au cours des cinq ans de suivi de leur étude. Cela a été motivé par les publications récentes de Shores et al., Araujo et al. et 
Laughlin et al. suggérant une corrélation directe entre taux de testostérone faible et mortalité. S'il y avait eu une telle corrélation dans leur étude, prendre en compte les sujets décédés au cours du suivi, qui risquaient d'avoir un taux de testostérone plus faible, aurait pu conduire à fausser l'estimation longitudinale des dépenses de santé liées au déficit en testostérone du fait de la possibilité de leur forte augmentation en fin de vie, puis de leur interruption à partir du décès. Cette exclusion a conduit à une augmentation du nombre estimé de consultations ambulatoires par patient et des coûts correspondants. Plus important, l'ajustement des données en fonction de l'existence d'un syndrome métabolique a abouti à des taux plus importants d'utilisation des structures de santé, tant en ambulatoire qu'en hospitalisation, lors de la réévaluation à cinq ans.

Il est de plus en plus évident qu'il existe un lien entre déficit en testostérone et syndrome métabolique, qui associe à une obésité viscérale une augmentation de la résistance à l'insuline et une production de facteurs inflammatoires en plus des anomalies métaboliques elles-mêmes. Cette étude suggère que la charge économique que constitue le déficit en testostérone via les systèmes de santé peut être accrue par son lien avec le syndrome métabolique, qui est par luimême une maladie très fréquente et coûteuse. Le syndrome métabolique semble donc un élément intermédiaire dans cette relation entre déficit en testostérone et augmentation de l'utilisation des systèmes de santé. Il est même probable que la durée de suivi relativement courte dans cette étude (cinq ans) a conduit à sous-estimer son rôle dans l'association testostérone basse-augmentation des dépenses de santé. Par contre, les autres pathologies associées telles que l'hypertension et le diabète ne semblent pas avoir joué de rôle dans cette association.
Limitations de cette étude : Le caractère relativement court du suivi (cinq ans), alors qu'une période plus longue aurait peut-être montré des conséquences à long terme plus importantes du déficit en testostérone. Par ailleurs, l'absence de mesure des fractions libres ou biodisponibles de la testostérone qui auraient peut-être permis une identification plus adéquate du déficit en testostérone.

Conclusions : En dépit de ces éventuelles limitations, les résultats de cette étude sont intéressants en ce qu'elle révèle l'importance du déficit en testostérone comme nouveau marqueur de la santé masculine et prédicteur du degré d'utilisation des systèmes de soin et des coûts générés par cette utilisation dans la population générale. Il faut cependant rappeler que comme il n'a pas encore été possible d'arriver à un agrément en ce qui concerne les limites à partir desquelles on peut parler de déficit en testostérone, les valeurs proposées actuellement restant arbitraires, et le diagnostic pouvant également dépendre des différentes méthodes de dosage, il est presque impossible d'estimer aujourd'hui l'impact économique réel du déficit en testostérone dans différentes populations. De toute façon, préciser le coût pour la santé du déficit en testostérone requiert d'établir des preuves plus importantes non seulement du lien physiopathologique qui unit déficit en testostérone et pathologies relatées, mais également de l'efficacité du traitement par la testostérone dans ces pathologies par l'intermédiaire d'essais cliniques en double insu contre placebo qui continuent de manquer. Ce n'est qu'à partir de ce moment qu'on pourra juger si traiter par la testostérone est une politique de santé rentable, compte tenu de la meilleure connaissance qu'on aura des conséquences à long terme du déficit en testostérone sur les dépenses de santé.

Conflit d'intérêt : aucun. 